\title{
Study of technological features of tubular compressed concrete members in concreting
}

\author{
Olena Voskobiinyk ${ }^{1 *}$, and Olga Gukasian ${ }^{1}$ \\ ${ }^{1}$ Poltava National Technical Yuri Kondratyuk University, Department of Civil Engineering, \\ Pershotravnevyi Avenue, 24, Poltava, 36011, Ukraine
}

\begin{abstract}
The technological features of core concreting were analyzed as the main factor in ensuring of strength and reliability of compressed concrete-filled steel tubular (CFST) members. We have conducted the analysis of existing concreting methods of CFST members. In this respect, the most dangerous types of possible technological defects of concrete core of CFST members are inhomogeneity along the height, voids, caverns, and concrete "weak spots". The authors considered the influence of such technological factors of concreting: placeability, time, concrete mixture compaction method, concreting height on the concrete core strength of CFST members. Based on the experimental studies conducted we suggested the regression correlations for determining the concrete strength of CFST members of different length depending on the movability of concrete mixture and a time for its compaction. The authors performed the correlation analysis of technological factors of concreting on the strength of the concrete core. We carried out the comparison of data on the concrete core strength of CFST members, that were determined by non-destructive methods (sclerometer test results, ultrasonic method) and direct compression strength tests. We experimentally proved that using movable mixtures with the slump of about $4-9 \mathrm{~cm}$ the overall variation coefficient of concrete core strength of CFST members along the height reaches nearly $13 \%$. Based on the experimental studies conducted we suggested the guidelines on optimal regimes of concrete compaction during manufacturing CFST members at a construction site environment.
\end{abstract}

\section{Introduction}

CFST elements are commonly used in global civil engineering practice. Their prevalence is justified by high strength characteristics, technological and economic indicators [1]. The modern state of the civil industry requires bearing structures to be a rational combination of high strength and low material consumption. CFST members meet the requirements completely.

Use of compressed CFST members as bearing structures is particularly important for industrial, residential and civil buildings. However, the known studies of CFST members $[2,3]$ are mostly dedicated to the study of its operational features under the load and to its stress-strain state.

\footnotetext{
* Corresponding author: elenvosko@,gmail.com
} 
Nevertheless, relevant literature summary [4] proves that the studies are primary focused on the characteristics and features of CFST members. These structures have the relatively small cross section area, but they can bear large weights. In these structures, due to the volumetric stressed state, concrete bears longitudinal force which is far beyond its prism strength. In previous surveys CFST members were tested in steel shell-tube with the diameter of 102 to $108 \mathrm{~mm}$ and the wall thickness of 3 to $3.5 \mathrm{~mm}$. Such specimens have a significant reinforcement percent. Steel shell-tube acts as external reinforcement and carries the load. A significant part of a load is born by steel part of a cross section of CFST members. It is hard to estimate the influence of concrete core defects (its size and location) on general strength of a specimen.

\section{Analysis of possible technological defects of CFST members concreting}

Known CFST members manufacturing methods divided into two groups: concreting of columns at the plant or directly at a construction site. From a technological point of view, when CFST members manufactured at a plant its concreting (filling in the shell-tube with concrete) could be carried out in vertical, horizontal and inclined positions.

When concreted inclined, the tubes placed at an angle of $25^{\circ}-30^{\circ}$ on the vibratory plate and filled with concrete mixture. Compaction carried out by vibration. The disadvantages here are the possibility of cavern and voids emerging which localized at the wall of shelltube. Among the advantages of this method, there is a possibility of using the heavy concrete mixtures (V4, S1).

Vertical concreting applied for manufacturing the CFST members with the length up to $5 \mathrm{~m}$. Concrete mixture supplied from the top, then it compacted by external vibrating. The disadvantage of this method is the possibility of concrete mixture separation when it supplied from a large height. It could cause the inhomogeneity of concrete structure along the height, as well as weak spots areas and incomplete compaction of a concrete mixture.

When horizontally concreted (centrifuging), the rotary form filled with concrete and compacted by rotation with the frequency of 900 rotations per second. Concrete mixture distributed across the form driven by centrifugal forces to the periphery of the form. Advantages of this method are high density and strength of concrete, low water consumption, permeability and increased resistance to aggressive influences. It is possible to concrete the tubes and other long items by centrifuge with low labor costs. The disadvantage of this method is the void with offset center and nonuniform concrete distribution on the walls (fluctuations in the thickness of the concrete layer).

CFST members concreting at a construction site carried out in three different ways: concreting the pack of tubes, concreting in design position and concreting through the perforation in a steel tube. Construction site concreting could cause the emerging of concrete core defects.

A pack of tubes fixed at an existing overlaying of a building and filled with movable concrete mixture out of the mixer. Specimens compacted by vibrating. One of the methods is a fixation of a depth-vibrator on the tube wall

When concreting the installed in design position tubes, concrete mixture supplied from the overlaying. Compaction of specimens made by external or deep vibrating. Tubes filled with concrete layer by layer, which could cause inhomogeneity of concrete features.

Concreting through a perforation in a tube carried out through the opening (with the diameter of $5 \mathrm{~cm}$ ) in shell-tube under the pressure. The advantage of this concreting method is an easy quality control process (it's possible to drill small holes with the diameter of less than $5 \mathrm{~mm}$ for control of filling the tube). The disadvantage of this method is the possibility 
of damaging the steel shell-tube. Moreover, the way of development of formwork defects couldn't be foreseen.

Manufacturing method and materials for CFST members have a major influence on its strength. Careful attention must be paid to the concreting quality. The concrete mixture has a significant variability of its physical and mechanical properties. Nonuniform distribution of the properties along the height could result in decreasing of carrying capacity for an entire structure.

Concrete non-homogeneity may be caused by a set of systematic and accidental factors [5]. These factors cause primary (natural), and secondary (virtual) non-homogeneity of features. True dispersion of concrete features caused by its natural non-homogeneity: variation of concrete components features, different compaction conditions, and other technological factors. Secondary non-homogeneity is an entropy due to the unclear method of sampling.

Analyses of CFST members manufacturing defects shows that the most significant ones are concreting anomalies (defects). These anomalies caused by technological complications which connected to the peculiarities of placing and compaction of concrete mixture into shell-tube inner space. Among them the most widespread there is caverns and voids formation at the contact surface between a core and a shell. These defects vary by the size, configuration and propagation depth in concrete body.

Accidental defects should also be contributed to technological violations of concreting. Among them, there are strength difference along the height of a specimen, concrete mixture separation, strength variability, non-homogeneity of structure and physical and mechanical features along the height of a specimen. Numerous surveys [6,7] show that separation could be avoided and homogeneity of a core could be reached even if the concrete mixture dropped from a significant $(10-15 \mathrm{~m})$ height during vertical concreting inside of a steel tube. Thereby, according to its genesis, the defects could be systemized in two groups: technological and accidental.

Concrete with a slump range from 6 to $16 \mathrm{~cm}$ recommended for filling in the CFST members (placeability marks S2, S3, and S4) [8]. Using lower values of concrete slump causes the defects of the concrete core. These defects are empty segments (voids and caverns at the tube wall) and inhomogeneity of strength along the height.

This research aimed at studying the influence of concreting technology on uniform distribution of strength (non-homogeneity of concrete strength along the height). As well as at studying the influence of vulnerable spots localization along the height of CFST members on its collapse character and strength.

\section{The methods of experimental researchers (Material and methods)}

We have conducted a set surveys in order to investigate the influence of technological factors on the core strength of CFST members. We used Box and Behnken experimental procedure of "3/1/15" type (table 1). It is equivalent to fractional factorial procedures $3 * *(k-p)$, but it also more economic due to applying the complex interaction effects. That allowed a minimum number of experiments to be conducted. According to the procedure, 15 experimental specimens were made of CFST members. To minimize the influence of force on resulting carrying capacity of CFST members we applied PVC tubes with the diameter of $109 \mathrm{~mm}$, filled with heavy concrete with $\mathrm{C} 20 / 25$ strength class $\left(f_{c m, c u l}=24.18 \mathrm{MPa}\right)$. We used polycarboxylates as a plasticizing additive for increasing the placeability of a concrete mixture.

During experiment planning, we investigated the influence of following factors: specimens height $\left(X_{l}\right)$, concrete mixture compaction time $\left(X_{2}\right)$ and placeability of a concrete 
mixture $\left(X_{3}\right)$. Each factor varied on three levels: " $-1 "$, " 0 ", “+1". Characteristics of specimens shown in table 1.

Table. 1. Procedure of experimental research of the concrete core strength of CFST members

\begin{tabular}{|c|c|c|c|c|c|c|c|c|c|}
\hline \multirow{2}{*}{$\begin{array}{c}\text { Specimen } \\
\text { cypher }\end{array}$} & \multicolumn{6}{|c|}{ Variable parameters and variation levels } \\
\cline { 2 - 11 } & \multicolumn{2}{|c|}{ Specimens height, mm } & \multicolumn{2}{c|}{ Compaction time, min } & \multicolumn{3}{c|}{ Placeability mark } \\
\cline { 2 - 10 } & -1 & 0 & +1 & -1 & 0 & +1 & -1 & 0 & +1 \\
\hline CFST-1(s) & 400 & & & 0 & & & V4 & \\
\hline CFST-2(s) & 400 & & & & & 4 & V4 & & \\
\hline CFST-3(m) & & 1200 & & & 2 & & V4 & \\
\hline CFST-4(1) & & & 2000 & 0 & & & V4 & & \\
\hline CFST-5(1) & & & 2000 & & & 4 & V4 & & \\
\hline CFST-6(s) & 400 & & & & 2 & & & S1 & \\
\hline CFST-7(m) & & 1200 & & 0 & & & & S1 & \\
\hline CFST-8(m) & 1200 & & & 2 & & & S1 & \\
\hline CFST-9(m) & 1200 & & & & 4 & & S1 & \\
\hline CFST-10(1) & & & 2000 & & 2 & & & S1 & \\
\hline CFST-11(s) & 400 & & & 0 & & & & & S2 \\
\hline CFST-12(s) & 400 & & & & & 4 & & & S2 \\
\hline CFST-13(m) & & 1200 & & & 2 & & & & S2 \\
\hline CFST-14(1) & & & 2000 & 0 & & & & & S2 \\
\hline CFST-15(1) & & & 2000 & & & 4 & & & S2 \\
\hline
\end{tabular}

During experiment planning, we investigated the influence of following factors: specimens height $\left(X_{1}\right)$, concrete mixture compaction time $\left(X_{2}\right)$ and placeability of a concrete mixture $\left(X_{3}\right)$. Each factor varied on three levels: " -1 ", " 0 ", “+1". Characteristics of specimens shown in table 1 .

All experimental specimens of CFST members hardened in the same environment. The specimens were cut into equal parts with the height of $l=2 d$ before the testing. Two short specimens made of each specimen marked "s", 6 short specimens made of each specimen marked " $\mathrm{m}$ " and 10 short specimens made of each specimen marked " $l$ ". The overall quantity of specimens was 90 units. Each one was measured, weighted and its strength was tested by three methods: non-destructive (using sclerometer and ultrasonic method) and destructive.

\section{Results and Discussion}

Results of research (figures 1 - 3) grouped by qualitative factor - initial length of the specimen (concreting height). Experimental data on the strength of specimen's component divided into three groups: 1 - specimens with the height of $400 \mathrm{~mm}$ (figure 1);2 specimens with the height of $1200 \mathrm{~mm}$ (figure 2); 3 - specimens with the height of 2000 $\mathrm{mm}$ (figure 3). Characteristic of concrete strength change with the height presented for each group.

First group - specimens with a height of $400 \mathrm{~mm}$ (figure 1). Concrete strength varies by $7 \%$. But the average strength of concrete of CFST $-1(\mathrm{~s})$ is the lowest $\left(f_{c m, c u l}=14.83 \mathrm{MPa}\right)$, it is $61 \%$ of the design strength. Compression strength decreasing of concrete is due to empty voids inside of it (diameter is $15 \mathrm{~mm}$, depth $15 \mathrm{~mm}, 8 \mathrm{~mm}, 7 \mathrm{~mm}$, and $4 \mathrm{~mm}$ ). Theses defects are caused by poor compaction. 


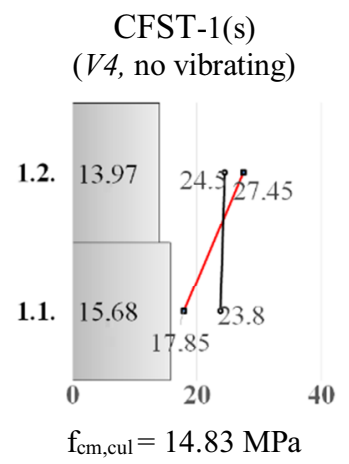

CFST-11(s)

( $S 2$, no vibrating)

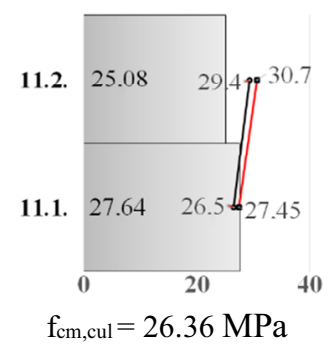

CFST-2(s)

$(V 4,4$ min.)

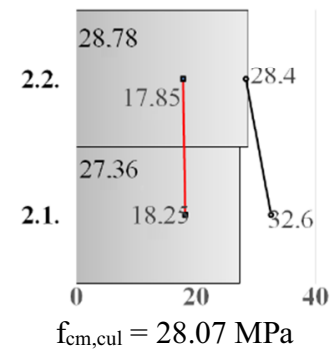

CFST-12(s)

(S2, 4 min.)

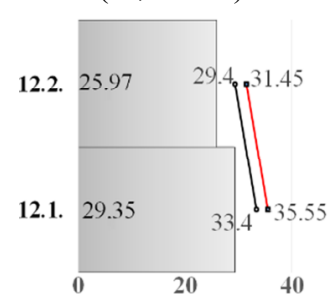

$\mathrm{f}_{\mathrm{cm}, \mathrm{cul}}=27.66 \mathrm{MPa}$
CFST-6(s)

(S1, 2 min.)

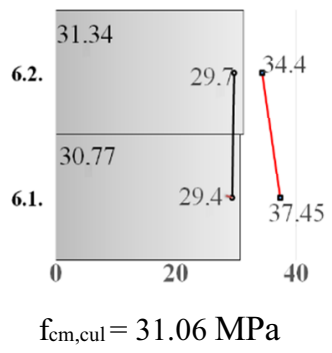

$\square$ Destructive method, Mpa;

$\rightarrow$-Sclerometer, MPa;

-o-Ultrasonic method, $\mathrm{MPa}$.

Fig. 1. Concrete strength variation along the height of CFST members with initial height of $400 \mathrm{~mm}$.

The voids discovered during specimen cutting. The average value of the strength is 2 times higher $\left(f_{c m, c u l}=28,07 \mathrm{MPa}\right)$ for the CFST-2(s) specimen. This one is of the same placeability mark but a compaction time was 4 minutes. That proves the necessity of using compaction for manufacturing the CFST from the stiff concrete mixtures. For all other specimens, the strength reserve varies from 9 to 23 percent. The average strength of specimens CFST-11(s) and CFST-12(s) is different by $5 \%$ under the same compaction time and concrete placeability mark.

Compression strength testing results of CFST-7(m) specimen are the lowest $\left(f_{c m, c u l}=14.83 \mathrm{MPa}\right)$. That proves that the absence of compaction decreased the concrete strength of CFST $-7(\mathrm{~m})$ specimen by $30 \%$. And CFST- $8(\mathrm{~m})$ specimen's average strength doesn't exceed $60 \%\left(f_{c m, c u l}=25.22 \mathrm{MPa}\right)$ compared to CFST-7 $(\mathrm{m})$.

The only difference here is the way of compaction - CFST-8(m) compacted by vibrating. The average value of its strength is in design limits. The only peculiarity of this specimen is decreasing of its strength at the top part. The difference in the technology of concreting CFST-8(m) and CFST-9(m) samples is compaction time. CFST-9(m) specimen (compaction time is 4 minutes) possesses the same strength of concrete along the height. Thereby, concreting defect (inhomogeneity at the top part) of CFST-8(m) specimen is the consequence of the lack of compaction. Specimens CFST-3(m) $\left(f_{c m, c u l}=27.17 \mathrm{MPa}\right)$ and CFST-9 $(\mathrm{m})\left(f_{c m, c u l}=27.24 \mathrm{MPa}\right)$ with almost the same average concrete strength are homogenous along the height. That proves a rational way of its manufacturing. Concrete strength along the height of CFST-13(m) specimen decreases two times from the bottom to the top. This caused by not rational compaction way. Comparing the results of testing of CFST members of medium height, there is an obvious stratification into 2-3 strength areas which emerge when the manufacturing methods are not rational

The second group is the specimens with the height of $1200 \mathrm{~mm}$ cut into 6 research fragments (figure 2). 


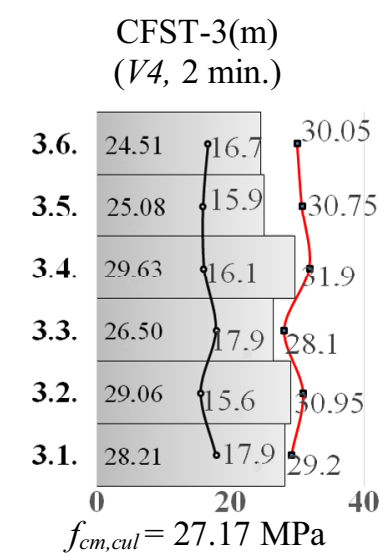

CFST-9(m)

(S1, 4 min.)

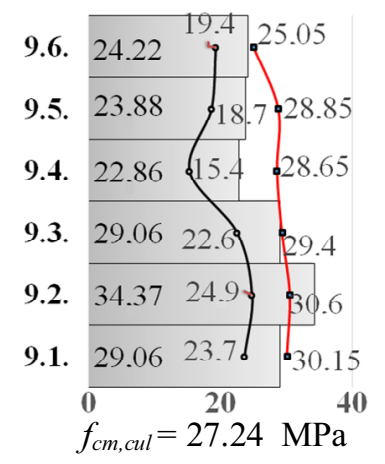

CFST-7(m)

(S1, no vibrating)

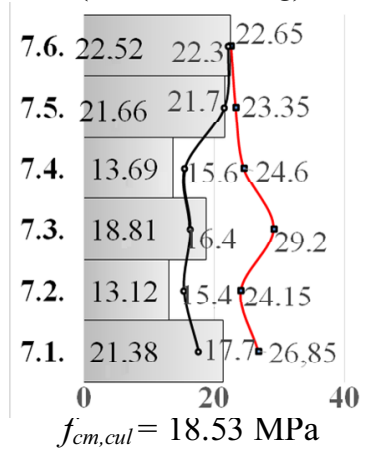

CFST-13(m)

(S2, 2 min.)

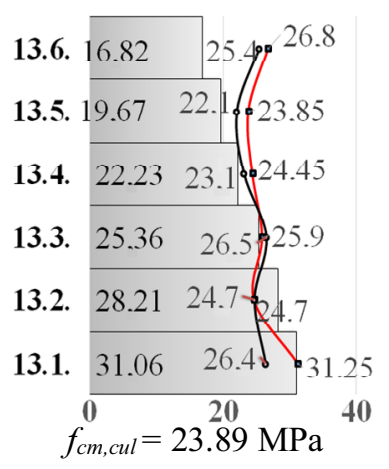

CFST-8(m)

(Sl, 2 min.)

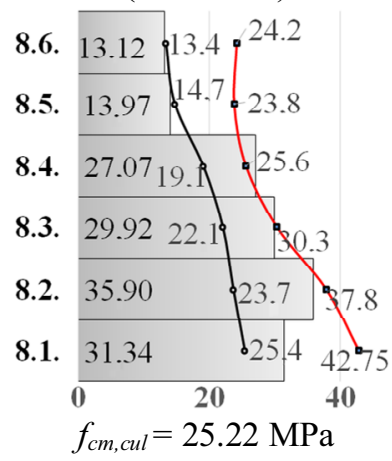

Measurement results by:

$\square$ Destructive method, Mpa;

$\rightarrow$-Sclerometer, MPa;

Ultrasonic method, $\mathrm{MPa}$.

Fig. 2. Concrete strength variation along the height of CFST members with initial height of $1200 \mathrm{~mm}$.

The third group is the specimens with the height of $2000 \mathrm{~mm}$ cut into 10 fragments (figure 3).

The average concrete strength of CFST-4 $(l)$ and CFST-5 $(l)$ decreased $(63 \%$ and $81 \%$ of design strength). CFST-5(l) specimen characterized by nonhomogeneous structure and concrete features along the height (strength along the height reduce in 3 times, a lot of concreting defects). Manufacturing conditions of CFST-4 $(l)$ and CFST-5(l) specimens are different in compaction way only. Concrete strength homogeneity along the height of CFST-4 $(l)$ specimen varies within the limits of $50 \%$. During concreting a long CFST members with V4 strength mark, defects couldn't be avoided irrespective of compaction. The average concrete strength of CFST member CFST-10 $(l)$ is $18 \%$ higher than calculated $\left(f_{c m, c u l}=28.71 \mathrm{MPa}\right)$. However, at this concreting method, the strength of specimen along the height is homogeneous, except for one fragment. Such spot of a high strength is likely to emerge during compaction (at the place of vibrating device location). The average value and homogeneity of CFST-14 $(l)\left(f_{c m, c u l}=24.75 \mathrm{MPa}\right)$ and CFST-15 $(l)\left(f_{c m, c u l}=25.25 \mathrm{MPa}\right)$ specimens is almost the same when the placeability mark of concrete is the same. The movable concrete mixture (the slump is $50-90 \mathrm{~mm}$ ) fills in even the small empty space completely without vibrating. There weren't any voids or caverns in CFST-14 $(l)$ and CFST$15(l)$ specimens.

Non-destructive control methods used to provide data for comparison with concrete compression tests results. They show the same dynamics of strength variation for homogeneous concrete structure. When there were some defects in concrete core, non- 
destructive control methods show increased strength values. Thereby, such control methods are not rational when applied to complete CFST members in case of possible defects of core concreting.

CFST-4 $(l)$

( $V 4$, no vibrating)

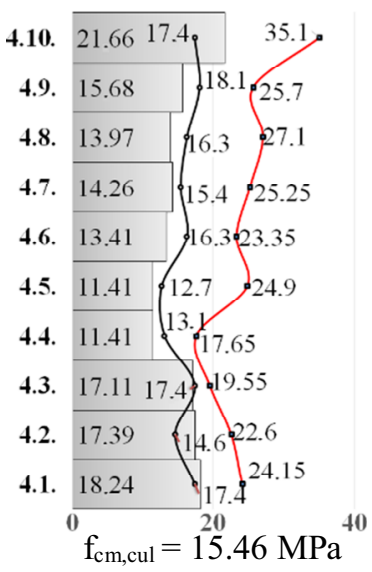

CFST-14 $(l)$

(S2, no vibrating)

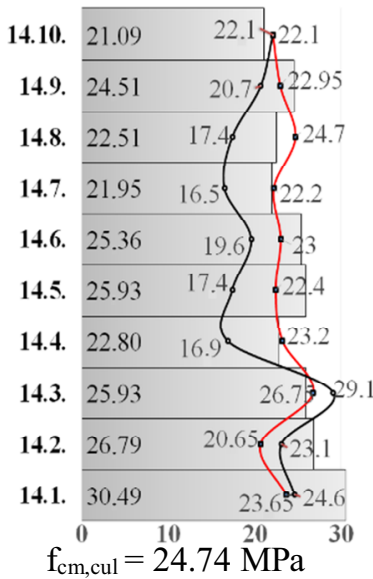

CFST-5(l)

(V4, 4 min.)

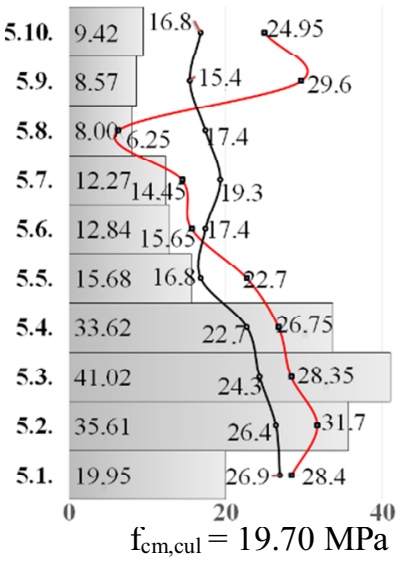

CFST-15(l)

$(S 2,4 \min$.

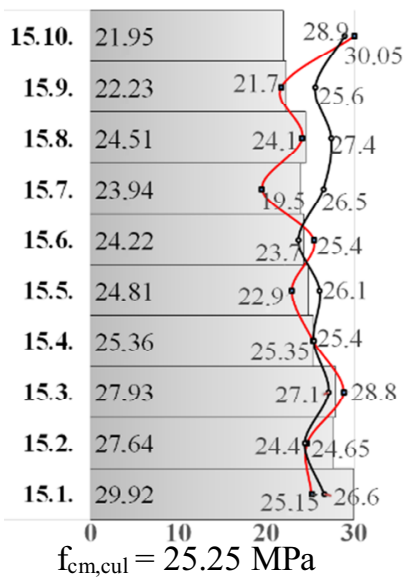

CFST-10 $(l)$

(S1, 2 min.)

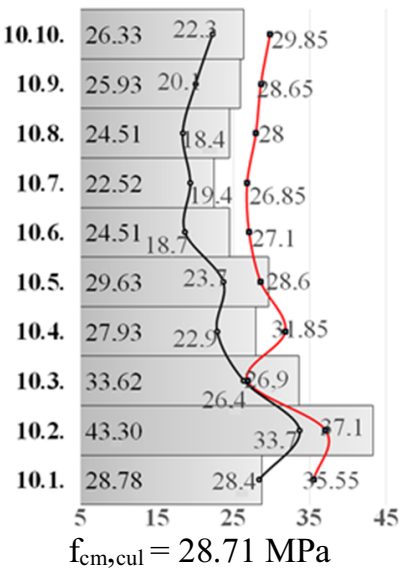

Measurement results by:

\section{$\square$ Destructive} method, Mpa;

$\rightarrow$-Sclerometer, MPa;

$\rightarrow$ Ultrasonic method, $\mathrm{MPa}$.

Fig. 3. Concrete strength variation along the height of CFST members with initial height of $2000 \mathrm{~mm}$.

According to research procedure, for an approximation of the obtained research data the following polynomial expression used:

$$
M_{u}=a_{o}+a_{1} x_{1}+a_{2} x_{2}+a_{3} x_{3}+a_{11} x_{1}^{2}+a_{22} x_{2}^{2}+a_{33} x_{3}^{2},
$$

Statistical data processing carried out using STATISTICA 10 software (figure 4). The obtained equations checked by Fisher criterion. Obtained regression equation allows estimation of the factors influence on the average compression strength of a specimen which calculated as an arithmetic average of all components (s $-2, \mathrm{~m}-6$ or $1-10$ values) for one specimen.

According to the result of statistical analysis, we established the correlations between variable factors and the strength. The optimal compaction time for short CFST members of 
"s" series is 2 minutes (figure 4, a). S2 placeability mark recommended for short specimens. Optimal compaction time is 4 minutes.

a) the influence of compaction time and specimen height on the compression strength
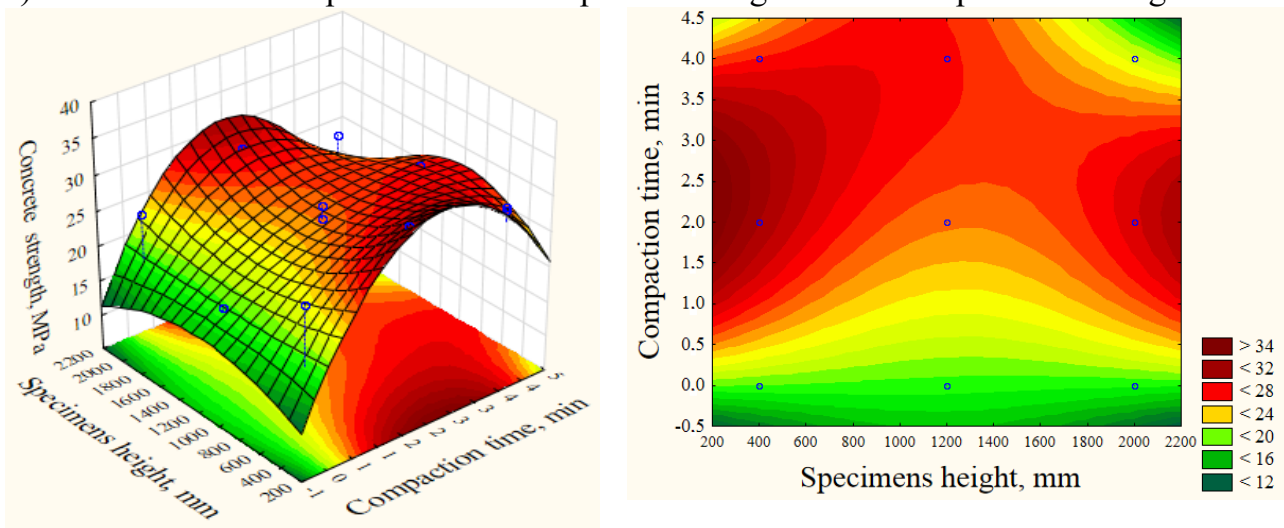

b) the influence of concrete placeability mark and the specimen height on the strength
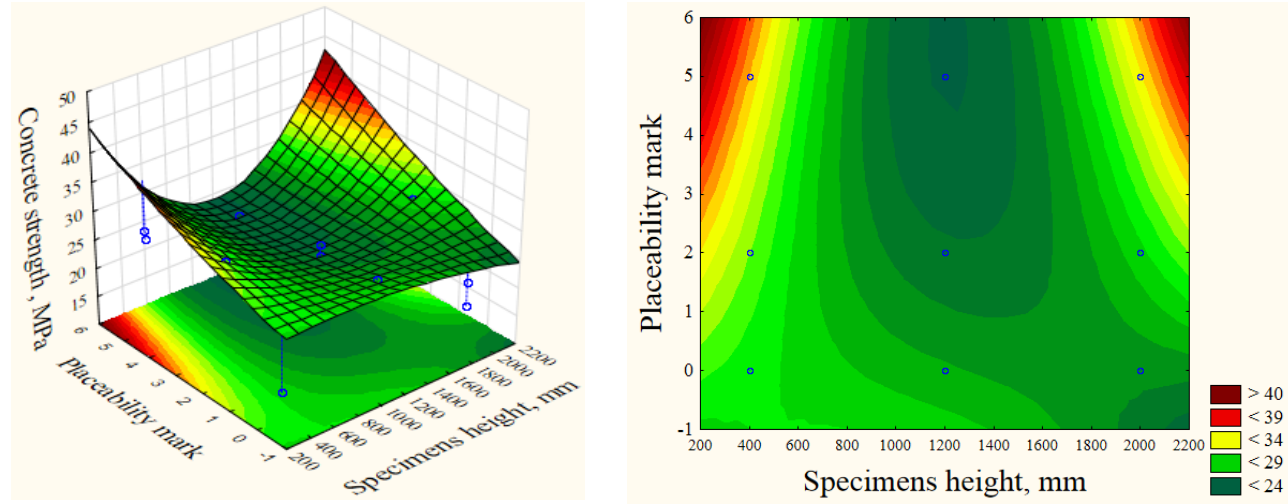

c) the influence of concrete placeability mark and compaction time on strength
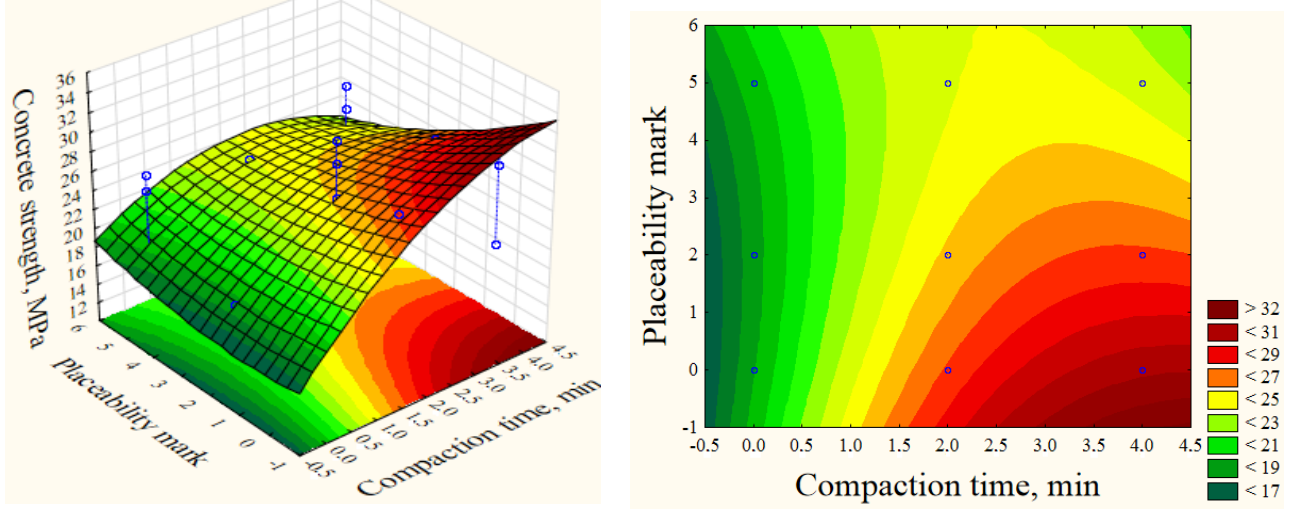

Fig. 4. Approximating surface of variation of the average value of concrete strength of CFST members in respect to experiment factors 
According to the result of statistical analysis, we established the correlations between variable factors and the strength. The optimal compaction time for short CFST members of "s" series is 2 minutes (figure 4, a). S2 placeability mark recommended for short specimens. Optimal compaction time is 4 minutes.

\section{Conclusion}

According to the developed method, we investigated the influence of concreting technology on strength distribution along the height of concrete core of CFST members.

Results of the research show that inconstancy of concrete strength along the height depends on placeability mark of the concrete mixture. Constant of variation of concrete core strength along the height of CFST members when stiff concrete mixtures used (V4) is 1.5...3.5 times higher than that of movable ones (S1 and S2).

Statistically significant correlation between the compaction time and concrete strength discovered when stiff mixtures used (V4) and movable mixtures with slump ranging from 1 to $4 \mathrm{~cm}$ (S1) 2 minutes of vibration time recommended as an optimal regime for concrete compaction. When movable mixtures with the slump of about $4-9 \mathrm{~cm}$ used, the total constant of variation of concrete strength along the height is about $13 \%$ irrespective of compaction time.

\section{References}

1. O.V. Semko, Probabilistic aspects of calculating composite constructions, Poltava (2004)

2. L.I. Storozhenko, Tube confined concrete, Kiev (1978)

3. L.I. Storozhenko, D.A. Ermolenko, O.I. Lapenko, Tube confined concrete, ASMI, Poltava, 306 (2010)

4. B. Uy, Z. Tao and L.H. Han, J CONSTR STEEL RES, 67, 360-378 (2011)

5. Leschynskyy A.M. Budivelnik, Kiev, 3, 47-49 (1986)

6. Kikin A.I., Sanzharovskii R.S. and Trul V.A., Constructions made of steel tubes filled with concrete, Stroyizdat, Moscow (1974)

7. Komar A.G., Technology of concrete and reinforced-concrete products, Stroyizdat, Moscow (1984)

8. Design of concrete structures - Part 1-1: General Rules and Rules for Bridges. EN 1992-1-1:2004 + AC:2010 Eurocode 2, Kiev (2012) 